\title{
Prenatal stress and childhood asthma risk: taking a broader view
}

\author{
Alison Lee ${ }^{1}$ and Rosalind J. Wright ${ }^{2,3}$
}

Affiliations: ${ }^{1}$ Division of Pulmonary, Critical Care and Sleep Medicine, Icahn School of Medicine at Mount Sinai, New York, NY, USA. ${ }^{2}$ Dept of Pediatrics, Kravis Children's Hospital, Icahn School of Medicine at Mount Sinai, New York, NY, USA. ${ }^{3}$ The Mindich Child Health and Development Institute, Icahn School of Medicine at Mount Sinai, New York, NY, USA.

Correspondence: Rosalind J. Wright, One Gustave L. Levy Place, Box 1198, New York, NY 10029, USA.

E-mail: rosalind.wrightamssm.edu

-

@ERSpublications

Cumulative evidence confirms stress in pregnancy is a critical prenatal risk for childhood asthma http://ow.ly/W1CCi

We now know that asthma risk begins in utero during rapid lung morphogenesis, when the developing fetus is particularly vulnerable to toxic insults due to immature immune, neuroendocrine and antioxidant defences. A meta-analysis published in a recent issue of the European Respiratory Journal (ERJ) [1] builds on substantial evidence highlighting psychological stress as a critical toxic exposure that, starting in utero, can permanently alter interrelated systems (i.e. immune, autonomic, neuroendocrine and oxidation systems) believed to programme lung growth and consequent respiratory disorders, including asthma [2].

Clinicians have long been challenged by the complexity of "what is asthma" and whether it is of the mind or the body. The concept that emotion plays a role in health is as old as medicine itself. References specifically linking psychological processes to asthma date back at least to the 12th century [3]. At the same time, one can find historical references from ancient Egypt and China on treating asthma by inhaling fumes from herb mixtures heated on bricks, including the shrub Ephedra (ma-huang) from which individuals inhaled beta-agonists [4]. As recently as the first half of the 20th century, Western medicine considered asthma to be psychogenic and treatment primarily involved psychoanalysis and other "talking cures" [5]. As science informed our understanding of the pathophysiological basis of airway inflammation and bronchial hyperresponsiveness, beginning in the 1960s, the psychiatric theory of asthma was largely set aside by the biomedical community, which then recognised asthma as a physical (i.e. inflammatory or allergic) condition [5]. Advances in psychoneuroimmunology shifted the paradigm once again and led scientists to reconsider the role of psychological stress in asthma, including the onset of disease [6].

A growing number of prospective epidemiological studies over the past decade have demonstrated significant associations between prenatal maternal stress and early asthma phenotypes in the next generation [7-11]. The magnitude of the association varies across these studies, probably due to differences in populations, study design, the stress measure used, characterisation of the outcome (e.g. wheeze or asthma), timing of exposure and adjusted confounders. In a recent issue of the ERJ, VAN DE Loo et al. [1] presented a well-designed meta-analysis, including 10 moderate to high quality studies (eight prospective cohort studies, one case-control study and one cross-sectional study), substantiating a significant relationship between prenatal stress and childhood asthma. The meta-analysis of both the crude and adjusted odds ratios from the included studies confirmed a significant association between increased prenatal maternal stress and childhood wheeze/asthma. In the adjusted meta-analysis, the overall pooled odds ratio of respiratory disease, including wheeze and asthma, among children born to mothers reporting higher stress (both stress exposure (i.e. experiencing a stressful event) and perceived stress (i.e. experiencing psychological symptoms or distress)) was 1.59 (95\% CI 1.3-2.0). Effects were strongest in the three

Received: Nov 172015 | Accepted after revision: Dec 092015

Conflict of interest: None declared.

Copyright OERS 2016 
prospective high-quality studies with a pooled adjusted odds ratio of 1.71 (95\% CI 1.3-2.3). The individual studies considered a range of important confounders, which included maternal age, education level and race/ethnicity, parental asthma or allergy, smoking during pregnancy, preterm birth, birth weight, the child's gender, and breastfeeding. The findings were similar in sensitivity analyses stratified by study quality, the approach to assessing stress, and the outcome being considered (wheeze, asthma or overall respiratory morbidity). Also, replication of findings in studies with populations that vary in age, geographic region and how the outcome was defined in this meta-analysis increases the generalisability of these findings.

Notably, the observed odds ratios may underestimate the true effect sizes in some of the studies included, as they adjusted for variables that may actually be in the pathway operating between maternal prenatal stress and asthma risk in the next generation. Mothers exposed to increased stress may be more likely to smoke prenatally and consequently at greater risk for preterm delivery and having a lower birth weight infant, factors that in turn have been linked to an increased risk of asthma development in early childhood. Including these pathway variables in the regression analyses may result in over-adjustment and an underestimation of true effects [12]. Future research in this area should consider mediation analyses to formally test such indirect effects in order to more fully understand the scope of the impact of stress and early asthma risk.

Knowing that the stress-asthma relationship has its roots in pregnancy is a critical beginning to the story, but in order to fully comprehend the impact of stress on respiratory health we need to take a broader view. Early effects may persist into adult life, magnifying health impacts, a concept known as the developmental origins of health and disease [13]. Stress exposure in early life not only effects wheeze/ asthma risk but also has implications for lung growth and development starting in utero [2]. We need to understand how stress exposures may be differentially associated with lung growth and development in early life as lung function patterns established early (i.e. by 7 years of age) track into adolescence and adulthood. Understanding how stress predisposes children to developing asthma is an important step in preventing disease and reducing respiratory-related morbidity and mortality over the life course.

Environmental exposures, including stress, operate during sensitive life periods to affect key physiological processes and regulatory systems that orchestrate development and the shaping of organ systems. As noted in a recent National Research Council workshop, research in humans has yet to elucidate the underlying molecular mechanisms or biomarkers that may identify those at heightened risk so that interventions may be applied early to promote optimal development [14]. Future studies should incorporate biomarkers of the interrelated systems (i.e. immune, autonomic, neuroendocrine and oxidation systems) hypothesised to be responsible for the association between maternal stress and child respiratory outcomes. Promising candidates that warrant focus include placental biomarkers [15], epigenetics [16-18] and developing neurobiological systems [19] that are responsive to stress as well as being implicated in respiratory health. Stress responsive mechanisms operating at the level of the placenta are only beginning to be elucidated and may provide markers of early risk [20]. Emerging studies demonstrate that different neurobiological systems are sensitive to stress exposure at different periods of development $[21,22]$. While the fetus is particularly vulnerable to stress due to immature immune, neuroendocrine and antioxidant defences, infants continue to be vulnerable as these systems are still developing and remain highly reactive and labile in response to environmental stressors in early life, particularly in the first 2 years.

Prenatal stress may also result in the altered programming of stress response systems, with enhanced vulnerability to subsequent stressful events such that those exposed in both time periods may be at greatest risk (i.e. a "two hit" model of disease). Pioneering animal studies demonstrate that effects of prenatal stress and/or hormonal correlates on offspring development may be different from those related to postnatal stress [19]. Other evidence demonstrates that an adverse postnatal environment modulates the developmental consequences of prenatal stress in a sex-specific manner. Sex-specific placental responsiveness to prenatal maternal stress and fetal sex hormones may contribute to differential effects of in utero stress on developmental outcomes. Proposed mechanisms include differential placental 11ß-hydroxysteroid dehydrogenase type 2 activity and/or sensitivity [23]; stress-induced oxidation in utero [24]; and/or prenatal stress effects on inflammatory disorders due to interactions of sex hormones and immune-inflammatory pathways [25]. Our group reported the first human data examining temporal- and sex-specific effects of stress in pregnancy and the early postnatal periods on wheeze in early childhood [26]. Specifically, boys were more sensitive to prenatal stress, while girls showed a stronger relationship between early postnatal stress and asthma risk. Future studies evaluating associations between maternal stress and child respiratory outcomes should include investigations into the sex-specific effects of both prenatal and postnatal caregiver stress. These studies will not only advance our understanding of sex differences in health and disease, but may begin to elucidate underlying mechanisms. Understanding basic mechanisms is an important first step in developing treatment strategies for stress-elicited asthma. For example, early caregiver-child interventions designed to normalise cortisol and autonomic nervous system responses may be effective in preventing asthma onset or reducing morbidity in those children with stress-elicited disease $[27,28]$. 
We also need to broaden our view to consider stress experienced over the mother's life course and not just the stress she experiences more immediately around or during pregnancy when characterising links between prenatal stress and child asthma. Even a woman's remote exposures to traumatic stressors such as violence and abuse may have particular influence on stress-related programming of respiratory disease that begins prenatally $[29,30]$. Psychological stress may also enhance the effects of other environmental toxins on asthma development and respiratory health, although studies in this area are sparse [31-33]. Thus, strategies to reduce stress exposure in key developmental periods may actually reduce the health consequences of other environmental toxins as well.

The effects of stress on childhood asthma risk can no longer be ignored. In addition to the research directions discussed earlier, efforts need to examine interventions focused on reducing stress in the prenatal and early postnatal periods that may reduce asthma development among all children. At the same time, we know that respiratory disorders such as asthma have significant sex differences in natural history, pathophysiology and response to treatment, which are not well understood. Prospective population-based studies assessing the complex interactions between stress, sex and developmental stage may more accurately identify health risks.

\section{References}

1 van de Loo KFE, van Gelder MMHJ, Roukema J et al. Prenatal maternal psychological stress and childhood asthma and wheezing: a meta-analysis. Eur Respir J 2016; 47: 133-146.

2 Wright RJ. Perinatal stress and early life programming of lung structure and function. Biol Psychol 2010; 84: $46-56$.

Rosner F. Moses Maimonides' treatise on asthma. Thorax 1981; 36: 245-251.

Cserhati E. The history of bronchial asthma from the ancient times till the Middle Ages. Acta Physiol Hung 2004; 91: 243-261.

5 Diamant Z, Boot JD, Virchow JC. Summing up 100 years of asthma. Respir Med 2007; 101: 378-388

6 Wright RJ, Rodriguez M, Cohen S. Review of psychosocial stress and asthma: an integrated biopsychosocial approach. Thorax 1998; 53: 1066-1074.

7 Mathilda Chiu YH, Coull BA, Cohen S, et al. Prenatal and postnatal maternal stress and wheeze in urban children: effect of maternal sensitization. Am J Respir Crit Care Med 2012; 186: 147-154.

8 Hartwig IR, Sly PD, Schmidt LA, et al. Prenatal adverse life events increase the risk for atopic diseases in children, which is enhanced in the absence of a maternal atopic predisposition. J Allergy Clin Immunol 2014; 134: 160-169.

9 Cookson H, Granell R, Joinson C, et al. Mother's anxiety during pregnancy is associated with asthma in their children. J Allergy Clin Immunol 2009; 123: 847-853.

10 Reyes M, Perzanowski MS, Whyatt RM, et al. Relationship between maternal demoralization, wheeze, and immunoglobulin E among inner-city children. Ann Allergy Asthma Immunol 2011; 107: 42-49.

11 Guxens M, Sonnenschein-van der Voort AM, Tiemeier H, et al. Parental psychological distress during pregnancy and wheezing in preschool children: the Generation R Study. J Allergy Clin Immunol 2014; 133: 59-67.

12 Schisterman EF, Cole SR, Platt RW. Overadjustment bias and unnecessary adjustment in epidemiologic studies. Epidemiology 2009; 20: 488-495.

13 Carraro S, Scheltema N, Bont L, et al. Early-life origins of chronic respiratory diseases: understanding and promoting healthy ageing. Eur Respir J 2014; 44: 1682-1696.

14 Boekelheide K, Blumberg B, Chapin RE, et al. Predicting later-life outcomes of early-life exposures. Environ Health Perspect 2012; 120: 1353-1361.

15 Jansson T, Powell TL. Role of the placenta in fetal programming: underlying mechanisms and potential interventional approaches. Clin Sci 2007; 113: 1-13.

16 McEwen BS, Bowles P, Gray JD, et al. Mechanisms of stress in the brain. Nat Neurosci 2015; 18: 1353-1363.

17 Krauss-Etschmann S, Meyer KF, Dehmel S, et al. Inter- and transgenerational epigenetic inheritance: evidence in asthma and COPD? Clin Epigenetics 2015; 7: 53.

18 Kabesch M, Michel S, Tost J. Epigenetic mechanisms and the relationship to childhood asthma. Eur Respir J 2010; 36: 950-961.

19 Bock J, Rether K, Gröger N, et al. Perinatal programming of emotional brain circuits: an integrative view from systems to molecules. Front Neurosci 2014; 8: 11.

20 Nugent BM, Bale TL. The omniscient placenta: metabolic and epigenetic regulation of fetal programming. Front Neuroendocrinol 2015; 39: 28-37.

21 Bosch NM, Riese H, Reijneveld SA, et al. Timing matters: long term effects of adversities from prenatal period up to adolescence on adolescents' cortisol stress response. The TRAILS study. Psychoneuroendocrinology 2012; 37: 1439-1447.

22 Constantinof A, Moisiadis VG, Matthews SG. Programming of stress pathways: a transgenerational perspective. J Steroid Biochem Mol Biol 2015 [in press; DOI: 10.1016/j.jsbmb.2015.10.008].

23 Mueller BR, Bale TL. Sex-specific programming of offspring emotionality after stress early in pregnancy. J Neurosci 2008; 28: 9055-9065.

24 Minghetti L, Greco A, Zanardo V, et al. Early-life sex-dependent vulnerability to oxidative stress: the natural twining model. J Matern Fetal Neonatal Med 2013; 26: 259-262.

25 Biswas DK, Singh S, Shi Q, et al. Crossroads of estrogen receptor and NF-kappaB signaling. Sci STKE 2005; 2005 : pe27.

26 Rosa MJ, Just AC, Tamayo y Ortiz M, et al. Pre- and postnatal stress and wheeze in Mexican children: sex-specific differences. Ann Allergy Asthma Immunol 2016 [in press; DOI: 10.1016/j.anai.2015.12.025].

27 Bernard K, Dozier M, Bick J, et al. Intervening to enhance cortisol regulation among children at risk for neglect: results of a randomized clinical trial. Dev Psychopathol 2015; 27: 829-841. 
28 Bosquet Enlow M, King L, Schreier HM, et al. Maternal sensitivity and infant autonomic and endocrine stress responses. Early Hum Dev 2014; 90: 377-385.

29 Sternthal MJ, Enlow MB, Cohen S, et al. Maternal interpersonal trauma and cord blood IgE levels in an inner-city cohort: a life-course perspective. J Allergy Clin Immunol 2009; 124: 954-960.

30 Schreier HM, Bosquet Enlow M, Ritz T, et al. Lifetime exposure to traumatic and other stressful life events and hair cortisol in a multi-racial/ethnic sample of pregnant women. Stress 2015 [in press; DOI: 10.3109/10253890.2015. 1117447].

31 Shankardass K, McConnell R, Jerrett M, et al. Parental stress increases the effect of traffic-related air pollution on childhood asthma incidence. Proc Natl Acad Sci USA 2009; 106: 12406-12411.

32 Islam T, Urman R, Gauderman WJ, et al. Parental stress increases the detrimental effect of traffic exposure on children's lung function. Am J Respir Crit Care Med 2011; 184: 822-827.

33 Peters JL, Cohen S, Staudenmayer J, et al. Prenatal negative life events increase cord blood IgE: interactions with dust mite allergen and maternal atoppy. Allergy 2012; 67: 545-551. 